\title{
Unidade de terapia intensiva: significados para pacientes em tratamento
}

\author{
Intensive care unit: meanings for patients in treatment
}

\author{
Maria do Carmo Eulálio ${ }^{a}$, Edivan Gonçalves da Silva Júnior ${ }^{b}$, Rafaella Queiroga Soutoc, \\ Lízie Emanuelle Eulálio Brasileiro ${ }^{\mathrm{d}}$ \\ a Psicóloga. Doutora em Psicopatologia Clínica pela Université Paul Valéry. Docente do curso de Psicologia da Universidade Estadual da Paraíba (UEPB). \\ b Psicólogo. Mestrando do Programa de Pós-Graduação em Psicologia da Saúde pela UEPB. \\ c Enfermeira. Doutora em Ciências da Saúde pela Universidade de São Paulo (USP). Docente do curso de enfermagem, na área de saúde pública da \\ Universidade Federal de Pernambuco (UFPE). \\ d Médica. Mestranda do Programa de Pós-Graduação em Ciências da Saúde pela Universidade Federal do Rio Grande do Norte (UFRN).
}

RESUMO Objetivo: O presente estudo teve como objetivo identificar e analisar os sentimentos suscitados por pacientes na Unidade de Terapia Intensiva (UTI), mediante a sua experiência de permanência nessa unidade.

Materiais e Métodos: Trata-se de um estudo qualitativo e descritivo. Participaram 25 pacientes internados em duas UTIs, com idades entre 15 e 85 anos. Entrevistas semiestruturadas foram realizadas e os dados foram submetidos à Análise Temática de Conteúdo.

Resultados: Destacaram-se as categorias: "Polaridade de sentimentos: positivos e negativos" e "Cuidado prestado pela equipe profissional".

Conclusão: Prevaleceram os sentimentos negativos relacionados principalmente ao medo que esta unidade hospitalar representa. A emergência de sentimentos positivos esteve direcionada à possibilidade de reestabelecimento do estado de saúde. Os cuidados e a atenção desenvolvidos pela equipe de intensivistas foram percebidos pelos pacientes como auxiliares no seu enfrentamento às dificuldades vivenciadas na UTI. Ressalta-se a importância da sensibilidade e da escuta às singularidades dos sujeitos em contato com a UTI.

Palavras-chave: sentimentos; pacientes internados; unidades de terapia intensiva. Materials and Methods: This is a qualitative descriptive study conducted with 25 patients from two ICUs, aged between 15 and 85 years old. Semi-structured interviews were conducted and the collected data were submitted to Thematic Contents Analysis. Results: These categories were highlighted: "Polarity feelings: positive and negative" and "Care provided by the professional team". Conclusion: The negative feelings related to the fear of what this hospital unity culturally represents prevailed. The positive feelings have been related to the possibility of the reestablishment of their healthy. Care and attention provided by the professional team were perceived by patients as an aid in coping the difficulties experienced in the ICU. Therefore, we emphasize the importance of sensitivity and listening to the singularities of people in the ICU.

Keywords: emotions; inpatients; intensive care units. 


\section{INTRODUÇÃO}

A Unidade de Terapia Intensiva (UTI) é considerada o espaço de maior complexidade técnica no hospital, uma vez que desempenha atividades centradas no cuidado de pacientes críticos, cujos quadros clínicos são marcados por grande instabilidade e possível risco de morte ${ }^{1,2}$. Em vista da intensidade das atividades desempenhadas nestas unidades, o espaço da UTI pode, então, apresentar-se como um dos ambientes mais agressivos, tensos e traumatizantes do ambiente hospitalar ${ }^{1}$.

Em decorrência da gravidade que envolve os quadros de pacientes que são tratados na UTI, estudos têm apontado que este ambiente acaba se tornando um espaço gerador de ansiedades, angústias, tristeza, estresse ${ }^{1,3,4,5,6}$. A emergência de tais sentimentos é compartilhada muitas vezes por profissionais ${ }^{7}$ e, principalmente, por familiares ${ }^{8}$ e pacientes $5,7,9,10$, o que contribui para o fortalecimento de crenças, significados e percepções que conferem a UTI um caráter mórbido e sombrio.

A estigmatização que recobre a UTI pode ser observada como resultado de um conjunto de significados culturais característicos deste ambiente. Fatores como o isolamento dos pacientes, a restrição às movimentações dentro da unidade, a rotina ininterrupta de atividades e o desconhecimento por parte dos pacientes sobre o seu estado atual de saúde são potencialmente geradores de insegurança e medo ${ }^{3}$.

A falta de conhecimento e de informação acerca dos serviços e atuações na UTI também acomete os pacientes ${ }^{5}$. A influência midiática que mantém imagens da UTI relacionadas diretamente à presença de tubos e sondas na boca e nas narinas dos pacientes, bem como a apresentação de ambientes frios e de solidão utilizada comumente para caracterizar a UTI pode contribuir para o desenvolvimento de sentimentos de medo em relação a esta unidade, facilitando o surgimento de fantasias de morte iminente para o paciente interno nesta unidade de saúde ${ }^{1}$.

Barlem et al. ${ }^{10}$ ressaltam que a internação do paciente na UTI inaugura uma mudança brusca nos seus modos de viver, repercutindo em interferências em suas relações, papéis e até mesmo em seus padrões de comunicação. A individualidade do paciente também está afetada em decorrência do seu processo de internação, uma vez que há a exposição de seu corpo, a ocorrência de barulhos excessivos, somados à falta de consideração de suas vontades e desejos ${ }^{11}$.

Com relação ao estado emocional que o paciente da UTI passa a experimentar, Rodrigues Jr e Amaral ${ }^{12}$ pontuam que em decorrência da situação de internação dentro de uma unidade intensiva tudo passa a ser decidido pelo outro, como a hora da alimentação, de tomar banho, de receber visitas e de ser manuseado. Como consequência, os pacientes que ali se encontram começam a demonstrar sentimentos bastante variados como angústia por estar só, medo do amanhã incerto, raiva por ser invadido em seu espaço físico e emocional sem a sua permissão diante de tamanha vulnerabilidade.

Observa-se, portanto, que os pacientes podem estar expostos a situações extremamente difíceis do ponto de vista emocional. Lucchesi et al. ${ }^{4}$, destacam que os desconfortos emocionais vivenciados na UTI podem evoluir para quadros de desestruturação emocional e até mesmo para o desenvolvimento de transtornos mentais reativos, a exemplo dos casos de transtornos de ajustamento, delirium, depressão, agitação psicomotora, episódios psicóticos, entre outros.

Tendo em vista o franco desenvolvimento das Unidades de Terapia Intensiva nos cuidados aos pacientes graves e da necessidade constante de preparação dos profissionais intensivistas que atuam nessas unidades para o contato direto com o sofrimento humano, cabe questionar como os pacientes vivenciam o ambiente da UTI. Quais os sentidos que os pacientes conseguem elaborar acerca de sua permanência nestas unidades? Que sentimentos conseguem expressar? Em resposta a tais questionamentos, objetivou-se identificar e analisar os sentimentos suscitados por pacientes na UTI, mediante a sua experiência de permanência nessa unidade.

\section{MATERIAIS E MÉTODOS}

Estudo descritivo, com abordagem qualitativa. A pesquisa desenvolveu-se em duas UTIs, uma em um hospital privado (Hospital I) que atende a convênios incluindo o Sistema Único de Saúde (SUS), e a outra UTI em um hospital (Hospital II) que atende exclusivamente o SUS. As duas unidades encontram-se em pleno funcionamento na cidade de Campina Grande, Paraíba.

A amostra foi do tipo não probabilística, por conveniência. As entrevistas foram realizadas com pacientes estáveis, internados em UTI. A quantidade de participantes que compõe a amostra foi definida pelo estado de saúde em que se encontravam os pacientes (que estivessem conscientes e orientados para aplicação das entrevistas), bem como pela livre aceitação dos mesmos em fazer parte do estudo durante o período de coleta de dados.

Os pacientes foram esclarecidos sobre os objetivos da pesquisa e questionados sobre a sua disponibilidade em participar da mesma. Foi solicitada a cada um dos participantes a assinatura do Termo de Consentimento Livre e Esclarecido, e garantido o sigilo das informações cedidas, bem como o direito de desistir a qualquer momento da pesquisa, sem quaisquer danos ao seu tratamento. 
A UTI do Hospital I atende ao SUS, convênios e particulares. Os principais serviços oferecidos por esta instituição são clínica médica, pediatria, ortopedia, cirurgia, ginecologia e obstetrícia. Na UTI adulto deste hospital há 10 leitos, dos quais dois são para pacientes com patologias infecto-contagiosas. Não há separação entre os sexos, nem por tipo de convênio. Em seus plantões, a unidade contava com um médico plantonista responsável, uma enfermeira, uma técnica em enfermagem e dois auxiliares.

A UTI do Hospital II atende exclusivamente a demanda do SUS. Entre os principais serviços oferecidos à comunidade estão o atendimento de emergência e urgência, clínica médica, ortopedia e neurologia. Nesta instituição, a UTI adulto é composta por nove (09) leitos, dos quais apenas um (01) é reservado para patologias infectocontagiosas. Os pacientes não são separados por sexo e idade, geralmente são separados dependendo do seu grau de patologia. A equipe profissional é composta por um médico plantonista, uma enfermeira, dois técnicos de enfermagem e três auxiliares. Embora direcionadas a atender a demanda do público adulto, as duas UTIs recebiam também pacientes advindos da pediatria, quando faltavam leitos para o atendimento nas unidades de referência a este público.

Para o desenvolvimento da pesquisa foram realizados um questionário demográfico e uma entrevista semiestruturada. A entrevista semiestruturada foi registrada com um gravador de áudio e posterior transcrição. O material produzido pelas entrevistas semiestruturadas foi analisado seguindo a técnica de Análise de Conteúdo Categorial Temática ${ }^{13}$. Para a autora, este tipo de análise consiste em desmembrar o texto em unidades, categorias segundo reagrupamentos analógicos. O critério utilizado foi à aproximação semântica, que permitiu categorizar o texto em temas mais específicos.

A pesquisa ocorreu em conformidade com a Resolução 466/2012 do Conselho Nacional de Saúde ${ }^{14}$, e aprovada pelo Comitê de Ética e Pesquisa da Universidade Estadual da Paraíba, sob o parecer - CAAE no 1095.0.133.000-05.

\section{RESULTADOS E DISCUSSÃO}

Houve predomínio do sexo masculino $(n=16 ; 64 \%)$. A idade dos participantes variou de 15 a 85 anos, com média de idade igual a 51,16 anos ( $D P=20,1)$. Quanto ao estado civil, predominaram pacientes casados $(n=15 ; 60 \%)$, seguidos de pacientes solteiros $(16 \% ; n=4)$, divorciados $(12 \% ; n=3)$, e viúvos $(12 \% ; n=3)$. De modo geral, os pacientes apresentaram baixa escolaridade, 20\% $(n=5)$ eram não alfabetizados, $36 \%(n=9)$ cursaram até o ensino fundamental, $24 \%(n=6)$ cursaram até o ensino médio e apenas $8 \%(n=2)$ alcançaram o ensino superior.
Para uma melhor compreensão acerca dos fatores que puderam contribuir mais significativamente para a experimentação dos sentimentos dos pacientes sobre a sua internação na UTI, desenvolve-se inicialmente uma breve explanação do seu tempo de internação nas duas unidades pesquisadas. Observou-se que $72 \%$ dos pacientes estavam internados há uma semana do período de realização da coleta, 20\% dos pacientes estavam internados há duas semana, enquanto que $8 \%$ dos pacientes se mantinham internados por três semanas.

A respeito do tempo de internação observado nos participantes, ressalta-se que o isolamento imposto por necessidades específicas da unidade, o pouco contato com os familiares e a ociosidade em ficar deitado todo o tempo no leito, podem levar os pacientes a ser tomados por sentimentos como medo de não sair com vida e ansiedade por presenciar ou ouvir casos de pacientes que não resistiram e faleceram, como também escutar diagnósticos que não conseguem compreender. Sendo assim, Kaplan et al. ${ }^{15}$ afirmam que a influência dos sentimentos sufocantes de medo pode acarretar no surgimento de sentimentos de apatia e indiferença, e até mesmo desencadear quadros de depressão.

Quanto ao estado de saúde dos participantes, buscou-se consultar a avaliação médica das condições gerais dos pacientes durante a realização da coleta de dados. Neste sentido, verificou-se que dez $(n=10 ; 24 \%)$ pacientes encontravam-se estáveis e nove $(n=9 ; 22 \%)$ esperavam alta da UTI. Oito $(n=8,22 \%)$ pacientes apresentavam um estado de saúde considerado bom pela equipe médica, enquanto que sete $(n=7,17 \%)$ pacientes apresentavam em estado de saúde regular, logo, requeriam maiores cuidados intensivos, no entanto, em condições de participação na pesquisa.

O processo de análise de conteúdo culminou na elaboração de duas categorias, importantes para a compreensão dos sentimentos e percepções dos pacientes: "Polaridade de sentimentos: positivos e negativos" e "Cuidado prestado pela equipe profissional".

\section{Categorias Temáticas}

\section{Polaridade de sentimentos: negativos e positivos}

\subsection{Sentimentos negativos}

Os sentimentos negativos associados a UTI despontaram com maior frequência entre os participantes, relacionados principalmente à invasão de um forte sentimento de medo e angústia frente ao suposto encontro do paciente com a morte. 
“Nunca pensei que viria pra cá. Pensei que ia voltar pra casa naquele dia mesmo. [...] Quando abri os olhos e vi o nome ali \{levanta um pouco a cabeça para mostrar o nome da unidade escrita na parede da UTI\} pensei que estava morto [...] e como pensei [...] quando olhei tudo isso e pensei que estava morto ou que ia morrer, mas a pessoa só morre no dia, né?" (Masc. 27 anos)

Através da fala do paciente é possível constatar que o medo da morte invade o seu pensamento. A respeito disso, Proença \& Dell Agnolo ${ }^{1}$ observaram em seu estudo que os pacientes trazem uma percepção prévia sobre a UTI, ligada essencialmente ao sentimento de insegurança e medo, característicos do estigma que é comumente atribuído a este setor. Observa-se, com isso, que no senso comum persiste a ideia associativa de que a internação na UTI pode caracterizar mais facilmente um perfil de pacientes à espera da morte, com poucas chances de cura ${ }^{1,3,5,8}$.

Outro sentimento aflorado pelos pacientes diz respeito ao medo da perda da sua independência e do controle de si próprio: "Porque até para tomar banho, tem que ficar pedindo ajuda [...] porque é ruim, ficar pedindo as coisas aos outros [...] Eu nunca imaginei estar na UTI não!" (Fem. 17 anos).

A perda do controle sobre o próprio corpo, assim como a perda do controle sobre a sua própria vida, afeta emocionalmente os pacientes que se veem abalados ao se depararem com a situação de dependência e restrições na $\mathrm{UTI}^{5}$. O sujeito se vê, então, desapropriado de sua condição de tomar decisões sobre si mesmo e daí pode resultar o sentimento de raiva e indignação por perceber-se invadido em seu espaço físico e emocional ${ }^{12}$, por ter a sua individualidade comprometida pela exposição do seu corpo ou pela desatenção aos seus desejos ${ }^{11}$.

Também se verificou o medo que o paciente possui de não sair de um quadro grave e, consequentemente, de não se curar de suas afecções. Este fato pode ainda ser reforçado quando os pacientes, ao se encontrarem conscientes nos leitos, observam aqueles que não conseguiram sobreviver em sua luta contra a doença.

Por estarem conscientes e ao mesmo tempo assustados com a rotina de trabalho da UTI, muitos pacientes mantém sua atenção no que acontece com o paciente ao lado, nos procedimentos que são realizados dentro da unidade. Por não entenderem a natureza dos procedimentos que são desenvolvidos acabam se agitando, ficam amedrontados ao escutar certas palavras que desconhecem e até mesmo ansiosos por verem pacientes em estados mais graves. A morte de um paciente é um evento chocante para aqueles que compartilham de um mesmo espaço de tratamento ${ }^{7}$.

A ausência da família na UTI foi percebida como uma grande falta para os pacientes, gerando um intenso sentimento de insegurança, uma vez que implica num corte com sua vida cotidiana, nos seus modos de interação. Trata-se de um período necessário de isolamento imposto pelo sistema administrativo das unidades hospitalares que é sentido negativamente pelos pacientes. Em se tratando desta situação, Backes et al. ${ }^{7}$ assinalam que ao se sentirem sozinhos, os pacientes relatam normalmente a falta que sentem dos seus familiares e compartilham o desejo de ter um acompanhante na UTI.

A atenção aos sentimentos de solidão e desamparo vivenciados frequentemente pelos pacientes merece conhecimento por parte da gestão hospitalar que deve pensar em estratégias de flexibilização e compensação das rotinas fixas que "engessam" o ambiente das unidades intensivas. Backes et al. ${ }^{7}$ mencionam os ganhos que familiares e pacientes percebem ao circularem em UTIs que possuem flexibilização na ampliação do número de horários de visitas e na duração destas, assim como quando é estimulada a interação dos pacientes entre si. Os autores defendem a necessidade de o paciente interagir nestes espaços, de manter relações com as outras pessoas, de modo a contribuir para que este possa sentir que a UTI não é necessariamente um lugar isento de comunicações. A UTI é um ambiente vivo, de luta e promoção da vida.

Com relação à experiência do tempo de estadia na UTI, os pacientes verbalizaram a sua insatisfação em passar por um longo tempo de internação, condição esta intensificada pelo fato de estarem sempre condicionados a permanecerem deitados, apenas olhando o teto e o movimento da unidade, escutando gemidos e gritos de dor dos demais pacientes, companheiros do mesmo espaço. Estes pacientes se veem numa difícil condição de espera de uma provável recuperação.

"A insegurança é pensar que estou sozinha [...] com dor nas costas, não posso mudar de posição, fico aflita, impaciente [...] tento dormir e não consigo nunca [...] acho que é o meu subconsciente falando alto [...] É porque eu sinto muito sono e ao fechar meus olhos, por mais que eu tente afastar, os pensamentos ruins ficam dentro da minha cabeça." (Fem. 33 anos)

"E como demora... A gente tem que ficar o tempo todo deitado assim! (fica duro na cama), olhando para o teto. Só pensando em outras coisas, vigiando, pra vê se o tempo passa mais depressa... Só Deus mesmo! [...] Eu viajo sim, me lembrando dos meus amigos, da minha infância [...] é o que me dá força [...] Que por mais que eu durma por causa dos remédios, o tempo não anda. [...] ai tem aquele relógio \{aponta para o relógio de parede\}, que por mais que eu tente não olhar pra ele, ele insiste em fica na minha frente." (Masc., 47 anos) 
As falas expressam condições prováveis de gerar estresse e ansiedade nos pacientes. Os fatores ambientais, tais como a luminosidade, os ruídos dos aparelhos e o desconforto nos leitos; as alterações nos ciclos circadianos, e a frequente intervenção de procedimentos invasivos contribuem para a instalação e agravamento do estresse nos pacientes, alertam Lucchesi et al. ${ }^{4}$. Em resposta a estas condições, faz-se necessário o levantamento de estratégias que contribuam para a redução dos estressores nas UTIs, em vista de promover uma melhoria nas condições de adaptação dos pacientes a este ambiente potencialmente estressor. Para tanto, deve-se levar em consideração os aspectos estruturais e funcionais deste setor. Trata-se de uma atividade possível de ser realizada, uma vez que, conforme apontam Rosa et al. ${ }^{6}$, a maioria dos estressores nas UTIs são passíveis de intervenções.

Observou-se também que a relação do tempo vivenciado pelos participantes na unidade de terapia intensiva é uma relação singular, uma vez que o tempo, segundo Eulálio ${ }^{16}$ :

[...] não é o tempo visto entre duas medidas de um relógio, mas como uma duração vivida. A temporalidade é designada como a consciência do tempo: ela é o tempo vivido [...] tempo subjetivo, afetivo, que marca uma impressão temporal no interior do sentimento. O sentimento é afetivamente elaborado e colocado em forma graças ao discurso, à narração que o sujeito faz (p. 77).

As percepções do passado, presente e futuro dão suporte para que os pacientes internados em UTI percorram um árduo caminho até o seu reestabelecimento.

\subsection{Sentimentos positivos}

Os sentimentos positivos identificados nos pacientes podem ser compreendidos, na situação de internação na UTI, quando os mesmos concedem uma boa "nota" a sua permanência nesta unidade, atribuindo que a sua passagem por ali lhes proporcionou uma possibilidade real de recuperação, através do cuidado especializado somado à força de vontade de sair e continuar a sua vida fora da instituição hospitalar. Esses sentimentos estão associados principalmente à esperança de continuar lutando contra a doença.

"Quando cheguei, pensaram \{a família\} que eu tava morta. [...] para o que eu tava \{quando deu entrada na UTI\}, tô bem demais. Só preciso ficar forte, pra poder andar de novo [...] nunca fiquei parada muito tempo [...] penso que pelo menos teria alguma chance pra sair e voltar a andar." (Fem., 75 anos)
Recorremos à Teoria Psicanalítica na sua referência aos mecanismos de defesa, com o intuito de compreender a ligação entre UTI e o surgimento de sentimentos positivos por parte dos pacientes. Destacamos, segundo esta perspectiva, que para dar vazão aos sentimentos inconscientemente negados, o ser humano adota o mecanismo de defesa. Dentre eles destaca-se a formação reativa, apontado por Ribeiro ${ }^{17}$ como um sentimento estritamente ligado ao sentimento da compensação. Trata-se, portanto, de uma defesa do ego contra impulsos agressivos e hostis, dos quais as situações de risco e eminência da morte, impulsionados pela internação na UTI, por exemplo, podem despertar como um possível mecanismo de defesa para o sujeito.

Ressalta-se a emergência do conceito de saúde elaborado pelos pacientes em seu confronto com a doença como um aspecto importante que sinaliza a presença de sentimentos de otimismo e esperança. A saúde foi vista como uma renovação, uma possibilidade de cura para os pacientes.

Discussões sobre o que venha a ser saúde e doença, quais os critérios normativos e até que ponto é abordado o defronte com a morte são questões que estão articuladas também à ideia de cuidado. Neste ponto, ressalta-se a necessidade de levantar esforços com o propósito de criar diferentes modos de tratamento e intervenção junto ao sujeito que adoece $^{18}$. Acolher os sentimentos negativos trazidos pelos pacientes e a demanda de suas concepções sobre a saúde e a doença constitui um importante meio de abrir espaços de expressão e de elaboração dos sentimentos vivenciados na UTI. Além disso, a atenção a estes aspectos pode culminar no fortalecimento das expectativas sobre a sua possibilidade de cura, e, consequentemente, podem resultar numa melhor adesão à terapêutica proposta nas unidades.

Assim, é importante considerar que apesar das frequentes reclamações dos pacientes acerca das inúmeras situações desconfortantes, mesmo diante da dependência causada pela gravidade de suas doenças, sobrepuja ainda o desejo pela sobrevivência, o desejo de ultrapassar a situação limite evidenciada pelo forte sofrimento vivenciado.

Alguns pacientes que já passaram por outra UTI permaneciam com medo da morte, mas ao mesmo tempo, por esta não ser mais uma realidade tão desconhecida, sabiam no mínimo que poderiam sair dali com vida e com uma melhor condição de saúde. Entende-se, portanto, que a experiência de cada paciente internado nesta unidade configura uma possibilidade singular de ressignificação de sua experiência.

\section{Cuidado prestado pela equipe profissional}

Os cuidados desenvolvidos pela equipe de intensivistas são essenciais para que o tratamento na UTI ocorra com qualidade e presteza suficiente para que o paciente possa 
acreditar na relevância do tratamento ao qual está sendo submetido ${ }^{2,7}$. Destarte, a internação hospitalar poderá funcionar como uma justificativa para a naturalização da segurança proporcionada pelos procedimentos, principalmente médicos, agentes capazes de operar mudanças significativas na reversão de quadros patológicos críticos para o reestabelecimento da saúde.

O núcleo temático que mais se destacou em relação à equipe profissional diz respeito aos sentimentos relacionados ao cuidado desenvolvido na UTI. O cuidado prestado pelos profissionais em forma de assistência e atenção aos pacientes se apresentou como uma importante justificava desenvolvida pelos pacientes ao julgarem a necessidade de sua estadia na terapia intensiva.

A consideração sobre os aspectos emocionais que envolvem os pacientes na UTI constitui uma forma de apreender necessidades que vão além da atenção que os aparatos tecnológicos, indispensáveis nos cuidados aos pacientes e no trabalho dos profissionais intensivistas, podem proporcionar como forma de cuidados à saúde. A respeito disso, Silva et al. ${ }^{11}$, discutem em sua pesquisa a importância da humanização nas unidades de terapia intensiva e acreditam que o fato de a equipe de enfermagem dispensar forte atenção à tecnologia, dedicando a esta o papel de principal responsável pelo cuidado e reestabelecimento dos pacientes graves, pode interferir na restauração da saúde nestas unidades.

Destarte, discute-se que os cuidados prestados por profissionais da saúde devem se fundamentar ao atendimento às necessidades humanas ${ }^{11}$, no resgate à sensibilidade através da empatia, de um compromisso moral ${ }^{2}$, de atenção aos fatores orgânicos, psicológicos e sociais; atitudes estas que denotam uma visão integral do sujeito.

A possibilidade de avaliar pacientes em hospitais públicos e privados mostrou-se importante na consideração dos sentimentos que são mais frequentes durante a permanência nas UTIs pesquisadas. Observou-se que os pacientes internados no Hospital I (que atende convênios e particulares, principalmente) evocaram mais frequentemente o tipo de assistência recebida, avaliada como satisfatória. Enquanto isso, os pacientes do Hospital II (atende exclusivamente a demanda do SUS) concentraram sua atenção nos aspectos negativos evidenciados pela própria doença e a saudade da presença dos seus familiares.

A boa assistência expressa pelos pacientes do Hospital I pode ser evidenciada a partir da seguinte fala: "Gostei de absolutamente tudo [...] da atenção, da assistência que se pode receber aqui dentro" (Masc., 63 anos).

Algumas pesquisas têm demonstrado que existem diferenças significativas entre os pacientes internados nas
UTIs de instituições hospitalares públicas e privadas. Em uma pesquisa realizada com pacientes de UTIs públicas e privadas foram encontradas diferenças significativas quanto às variáveis idade, mortalidade e gravidade, apontando para uma maior frequência destas duas últimas variáveis nos pacientes das instituições públicas ${ }^{19}$. O fato pode ser reforçado através da percepção de enfermeiros intensivistas que relatam em sua prática clínica a necessidade de uma maior demanda de cuidados dispensados a pacientes internados em UTI de hospitais públicos, quando comparados aos pacientes de unidade crítica privada ${ }^{20}$. Visto de outro lado, sob o ponto de vista dos pacientes, a presente pesquisa demonstra que estes também apresentam sentimentos diferentes nas duas instituições, na qual a percepção sobre o cuidado prestado pode ser tido como mais evidente na instituição privada.

Ademais, foi possível observar o sentimento de insegurança presente nos pacientes desencadeado principalmente pelo fato de não haver ninguém que os informasse sobre sua real condição de saúde, informações que correspondiam à necessidade de um determinado prognóstico, principalmente positivo. Esta pode ser considerada uma situação vivenciada sob o "reino" da submissão e dependência, muitas vezes total, a uma equipe especializada em casos graves.

A respeito da necessidade deste diálogo, os pacientes foram indagados sobre como ocorria a comunicação durante sua internação na UTI. A partir disso foi possível perceber uma diferença entre o procedimento nos dois hospitais pesquisados: no Hospital I, a frequência do relato de comunicação pelo próprio médico foi alta, enquanto que no Hospital II, a maioria dos pacientes só soube da internação na própria UTI. Estes últimos relatavam que recebiam informações apenas da equipe de enfermeiros intensivistas.

De acordo com Barlem et al. ${ }^{10}$ a comunicação desenvolvida pela equipe profissional na UTI se faz essencial para que o paciente possa se situar naquele que se configura muitas vezes como um ambiente impactante e desorientador, marcado pelo forte sentimento de inconformismo. Os autores defendem o exercício da comunicação como um importante instrumento de cuidado, no qual a manifestação verbal e não verbal podem estimular reações nos pacientes, podendo ajudá-los na superação de seus problemas, na facilitação de seus relacionamentos dentro da UTI e, consequentemente, no ajustamento a situações que muitas vezes não poderiam ser modificadas.

Neste sentido, resgata-se, em meio a uma série de fatores que contribuem para uma percepção negativa da unidade de terapia intensiva, o sentido do cuidado humano. Backes 
et al. ${ }^{9}$ afirmam que o cuidado, compreendido como um fenômeno complexo, porta um sentido integrador, capaz de "acolher e respeitar os valores, as crenças, a cultura e as expectativas de vida que são singulares a cada ser humano, como paciente, familiar ou profissional".

\section{CONSIDERAÇÕES FINAIS}

Observou-se que os sentimentos positivos demonstraramse povoados em suas ideias sob a forma de esperança, da possibilidade de continuar persistindo em reestabelecer um estado anterior de saúde. Por outro lado, o sentimento de morte representa um perigo real devido à experiência vivenciada pela súbita internação, ou mesmo pela representação que acompanha os pacientes mesmo antes de se internarem na UTI, relativa à associação imediata desta unidade ao sentimento iminente de morte. Desta forma, o pensamento dos pacientes internados nesta unidade é povoado por um sentimento socialmente compartilhado, de aproximação com a morte, o que gera fortes sentimentos de ansiedade e angústia.

Em contrapartida a todo o sofrimento gerado, intensificado pela condição de fragilidade física, principalmente, foi possível observar que o apoio da família, as boas lembranças que os pacientes conservam, sua esperança e força de vontade podem atuar como importantes mecanismos de enfrentamento às problemáticas vivenciadas, auxiliando na redução dos estressores que cada paciente percebe no ambiente da unidade intensiva. A observação a estas condições, revistas sobre a sensibilidade da equipe de profissionais intensivistas, pode resultar numa estratégia de fortalecimento dos pacientes no enfrentamento às adversidades causadas pela gravidade de suas doenças, ajudando-os a suportar melhor a dor, a solidão e o próprio sentimento de dependência e submissão ao cuidado de terceiros.

Com relação aos dois tipos de atenção dispensados aos pacientes intensivos, ressalta-se a necessidade de os profissionais intensivistas atentarem para a dimensão afetiva que a prática do cuidado exige, mesmo quando o trabalho na UTI demanda dedicação de grande parte do seu tempo aos cuidados práticos, imprescindíveis para estabilização dos quadros patológicos dos pacientes graves.

Em última análise, pode-se resumir o conjunto das necessidades percebidas através do respeito à singularidade de cada paciente, possível de ser executado a partir do exercício de um cuidado humanizado, de valorização da comunicação da equipe profissional com os pacientes e da estimulação de formas de interação num espaço em que deve ser reconhecido devidamente o seu caráter incondicional de luta pela vida.

\section{REFERÊNCIAS}

1. Proença $M O$, Dell Agnolo $C M$. Internação em unidade de terapia intensiva: percepção de pacientes. Rev Gaúcha Enferm. 2011;32(2):279-286. http://dx.doi.org/10.1590/S1983-14472011 000200010

2. Silveira, RS, Martins CR, Lunardi VL, Vargas MAO, Filho WDL, Avila LI. A dimensão moral do cuidado em terapia intensiva. Cienc Cuid Saude. 2014;13(2):327-34. http://dx.doi.org/10.4025/ cienccuidsaude.v13i2.19235

3. Lemos, RCA, Rossi LA. O significado cultural atribuído ao centro de terapia intensiva por clientes e seus familiares: um elo entre a beira do abismo e a liberdade. Rev Latino-am Enferm. 2002;10(3): 345-57. http://dx.doi.org/1590/S0104-11692002000300009

4. Lucchesi F, Macedo PCM, De Marco MA. Saúde mental na unidade de terapia intensiva. Rev SBPH. 2008;11(1):19-30.

5. Pina RZ, Lapchinsk LF, Pupulim JSL. Percepção de pacientes sobre o período de internação em unidade de terapia intensiva. Cienc Cuid Saude. 2008;7(4):503-8. http://dx.doi.org/10.4025/cienccuidsaude. v7i4.6658

6. Rosa BA, Rodrigues RCM, Gallani CBJ, Spane TM, Pereira CGS. Estressores em unidade de terapia intensiva: versão brasileira do The Environmental Stressor Questionnaire. Rev Esc Enferm USP. 2010;44(3):627-35. http://dx.doi.org/10.1590/S008062342010000300011

7. Backes MTS, Erdmann AL, Büscher A. O cuidado intensivo oferecido ao paciente no ambiente de unidade de terapia intensiva. Esc Anna Nery. 2012;16(4):689-96. http://dx.doi.org/10.1590/S141481452012000400007

8. Maestri E, Nascimento ERP, Bertoncello KCG, Martins JJ. Avaliação das estratégias de acolhimento na unidade de terapia intensiva. Rev Esc Enferm USP. 2012;46(1):75-81. http://dx.doi.org/10.1590/ S0080-62342012000100010

9. Backes MTS, Backes DS, Erdamann AL. Relações e internações no ambiente de cuidados em unidade de terapia intensiva. Acta Paul Enferm. 2012;25(5):679-85. http://dx.doi.org/10.1590/S010321002012000500006

10. Barlem ELD, Rosenhein DPN, Lunardi VL, Lunardi Filho WD. Comunicação como instrumento de humanização do cuidado de enfermagem: experiências em unidade de terapia intensiva. Rev Eletr Enf [Internet]. 2008 [cited 2015 Jan 10];10:[8 telas]. Available from: http://www.fen.ufg.br/revista/v10/n4/v10n4a16.htm

11. Silva FD, Chernicharo IM, Silva RC, Ferreira MA. Discursos de enfermeiros sobre humanização na unidade de terapia intensiva. Esc Anna Nery. 2012;16(4):719-27. http://dx.doi.org/10.1590/ S1414-81452012000400011

12. Rodrigues Jr GR, Amaral JLG. Impacto psicológico da internação na unidade de terapia intensiva. Rev Bras Ter Intensiva. 2001;13(4): 92-98.

13. Bardin L. Análise de conteúdo. São Paulo: Edições 70; 2011.

14. Conselho Nacional de Saúde (BR). Resolução no 466, de 12 de dezembro de 2012. Brasília: CNS; 2012.

15. Kaplan RI, Sadock BJ, Grebb JA. Compêndio de psiquiatria: ciência do comportamento e psiquiatria clínica. 9th ed. Porto Alegre: Artemed; 2007. 
16. Eulálio MC. A história oral: vetor tempo para pessoas idosas. In: Whitaker DCA, Velôso RMG, organizadoras. Oralidade e subjetividade. Campina Grande: EDUEP; 2005. p. 69-85.

17. Ribeiro JP. Teorias e técnicas psicoterápicas. Petrópolis: Vozes; 2013.

18. Czeresnia D, Maciel EMGS, Oviedo RAM. Os sentidos da saúde e da doença. Rio de Janeiro: Editora Fiocruz; 2013.

19. Silva E, Pedro Mde A, Sogayar AC, Mohovic T, Silva CL, Janiszewski M, Cal RG, de Sousa EF, Abe TP, de Andrade J, de Matos JD, Rezende
E, Assunção M, Avezum A, Rocha PC, de Matos GF, Bento AM, Corrêa AD, Vieira PC, Knobel E; Brazilian Sepsis Epidemiological Study (BASES study). Crit Care. 2004;8(4): R251-60. http://dx.doi. org/10.1186/cc2892

20. Nogueira LS, Koike KM, Sardinha DS, Padilha KG, Sousa RM. Carga de trabalho de enfermagem em unidades de terapia intensiva públicas e privadas. Rev Bras Ter Intensiva. 2013;25(3):225-32. http://dx.doi.org/10.5935/0103-507X.20130039 